\title{
Core-Shell Bimetallic Au-Pd Nanoparticles: Synthesis, Structure, Optical and Catalytic Properties
}

\author{
P. Venkatesan*, J. Santhanalakshmi
}

Department of Physical Chemistry, University of Madras, Guindy Campus, A.C.Tech., Chennai, Tamil Nadu, 600025, India

\begin{abstract}
Colloidal dispersions of bimetallic nanoparticles composed of gold and palladium were prepared by wet chemical method, in which $\mathrm{Au}(\mathrm{III})$ and $\mathrm{Pd}(\mathrm{II})$ ions in an aqueous solution in the presence of a cationic surfactant, Cetyltrimethylammonium bromide (CTAB). The structure and composition of the metallic nanoparticles were characterized by UV-visible spectroscopy (UV-Vis), High-resolution transmission electron microscopy (HRTEM), Scanning electron microscopy (SEM-EDX), X-ray diffraction (XRD), X-ray photoelectron spectroscopy (XPS) and Fourier transform infrared spectroscopy (FTIR). Transmission electron microscopy photographs showed spherical particles whose size had a fairly narrow distribution with a geometric mean diameter about $9.4 \mathrm{~nm}$. Analyses with UV-Vis spectra indicated that $\mathrm{Au}(\mathrm{III})$ ions were first reduced and after their consumption reduction of Pd(II) ions set in. A core-shell structure of the particles, a core of gold and a shell of palladium was confirmed by high-resolution TEM and X-ray diffraction. The catalytic activities of nanoparticles are tested on the hydrogenation reaction between $\mathrm{NaBH}_{4}$ and 4-Nitrophenol reduction was studied spectrophotometrically. Bimetallic nanoparticles exhibited better catalysis that the mono metallic nanoparticles, which may be due to the electronic effects of the core to shell metal atoms.
\end{abstract}

Keywords Gold Nanoparticle, Palladium Nanoparticle, Gold-Palladium Bimetallic Nanoparticles, Hydrogenation, 4-Nitrophenol, Kinetic Study

\section{Introduction}

Bimetallic nanoparticles exhibit interesting electronic, optical, and chemical or biological properties due to new bi-functional or synergistic effects[1-10]. Among the chemical characteristics of metal nanoparticles, their catalytic property is of great interest and is the best investigated [11-13]. The particle size can affect not only the activity but also the selectivity of catalysts. Consequently, metal nanoparticles have provided new opportunities for catalysis and research activities in this area have paved the way to new solid catalysts over the past decade. From the catalytic point of view, bimetallic nanoparticles, composed of two different metal elements, have drawn a greater interest than monometallic ones because bimetallization would make it possible not only to obtain an improved catalytic activity but also to create a new property, which may not be achieved by monometallic catalysts[14]. However, Gold and palladium nanoparticles[15-18] have recently emerged as viable catalysts of a wide range of interests. The formation of surfactant supported gold nanoparticles or gold-containing bimetallic nanoparticles are expected to enhance the catalytic activity

* Corresponding author:

venkatesanorg@gmail.com (P. Venkatesan)

Published online at http://journal.sapub.org/nn

Copyright $(\underset{2}{ } 2011$ Scientific \& Academic Publishing. All Rights Reserved and selectivity[19-21]. Several types of gold-based bimetallic nanoparticles for catalytic reactions have recently been studied in our laboratory[22]. Gold-palladium (Au-Pd) nanoparticles and their assemblies have shown interesting catalytic and structural properties. Recently, $\mathrm{Au}-\mathrm{Pd}$ nanoparticles of certain bimetallic compositions have been synthesized at different methods[23-29]. The synthesis of the bimetallic nanoparticle system with composition, size controllability and core-shell structure of nanoparticles in a wide range has not been established. This situation is in part due to the fact that there is the propensity for $\mathrm{Pd}^{2+}$ to form precipitation with halogen ions in aqueous solutions when it is used as a precursor in combination with $\mathrm{AuCl}_{4}$ - precursor and the application of these bimetallic nanoparticles as catalysts [30-32].

In this paper we demonstrate that the successive reduction of $\mathrm{Au}$ and $\mathrm{Pd}$ salts can lead to the formation of core-shell Au-Pd nanoparticles, which display interesting optical properties. The palladium nanoparticles changes progressively as new layers are deposited, but an approximately concentric geometry can be readily maintained. Additionally, we present preliminary XPS and XRD results, which seem to indicate that reduction of $\mathrm{Au}$ on $\mathrm{Pd}$ surfaces leads to formation of a core-shell structure of the nanoparticles. The catalytic activities of nanoparticles are tested on the hydrogenation reaction between $\mathrm{NaBH}_{4}$ and 4-Nitrophenol reduction was studied by spectrophotometrically. 


\section{Experimental}

\subsection{Preparation of Au-Pd Bimetallic Nanoparticles}

Our laboratory recently developed a methodology for sequential reduction and preparing mono and bimetallic core-shell nanoparticles by wet chemical method of mixtures consisting two metal colloids. This approach is based on the melting behavior of colloids under this chemical method, which leads to core-shell particles. In view of our successful preparation of various mono and bimetallic nanoparticles [22], we took the next step and conducted the first trial of simultaneous reduction of two metals in chemical methods in $\mathrm{Au}-\mathrm{Pd}$ colloidal mixtures. The colloidal dispersions of surfactant protected Au-Pd core-shell bimetallic nanoparticles were prepared by refluxing of the aqueous solution of $\mathrm{HAuCl}_{4}$ and $\mathrm{PdCl}_{2}$ in the presence of CTAB. The molar ratio of Au-Pd was 1:1 and the molar ratios of monomer unit of $\mathrm{CTAB}$ against total metal (R) were kept 30 in the present experiments. The colloidal dispersion of CTAB-protected $\mathrm{Au}-\mathrm{Pd}$ (1:1) precursor metal salts are mixed at refluxed temperature in the designated ratio at one hour. The mixed dispersions are kept stirring at least for a $24 \mathrm{hrs}$ at room temperature to complete the self-organization reaction. The bimetallic with different compositions molar ratio (1:0.5 and $0.5: 1)$ of the precursor metal salts of gold and palladium could be prepared without any precipitation. The solutions of all the compositions are very stable over extended periods of time.

\subsection{Catalytic Study}

To investigate the catalytic reduction of 4-Nitrophenol, $0.5 \mathrm{~mL}$ of solution containing magnetically recoverable mono metallic Au or Pd or bimetallic Au-Pd core-shell nanoparticles (an equal amount of nanoparticle was used) catalyst was mixed with 4-Nitrophenol and the volume of the mixture was adjusted to $10 \mathrm{~mL}$ with double-deionized water. Then $5 \mathrm{~mL}$ of $\mathrm{NaBH}_{4}$ (cold solution) solution was rapidly added under constant stirring. The yellow color of solution gradually vanished, indicating the reduction of 4-Nitrophenol. The reduction concentration of 4-Nitrophenol was determined using Shimadzu UV-1601 double beam spectrometer using quartz cuvettes with $1 \mathrm{~cm}$ path length was used for UV-Vis spectra. After the completion of the reaction, $0.1 \mathrm{M} \mathrm{HCl}$ solution and 4-fold excess of $\mathrm{CH}_{2} \mathrm{Cl}_{2}$ are added so that, the mono or bimetallic nanoparticles catalyst and the products are correspondingly separated into the $\mathrm{pH}$ responsive aqueous and organic phases respectively. The products in the organic solvent are purified by chromatography on silica gel and are analyzed by NMR and GCMS data. The precipitate of $\mathrm{pH}$-responsive metal nanoparticles catalyst was collected by ultracentrifugation. The collected catalyst is dried and reused for the next cycle of reduction reaction.

\section{Results and Discussion}

Figure 1, present the UV-Vis spectra of mono metallic
$\mathrm{Au}, \mathrm{Pd}$ and bimetallic Au-Pd nanoparticles showing the SPR peaks respectively. Figure 2 shows a representative TEM image of the surfactant stabilized mono and bimetallic nanoparticles. It can be seen that the mono and bimetallic nanoparticles are mono disperse with an average size of 5.6, 6.0 , and $9.2 \pm 0.4 \mathrm{~nm}$ corresponding to mono metallic ( $\mathrm{Au}$, $\mathrm{Pd})$ and bimetallic (Au:Pd, 1:1) nanoparticles respectively, the TEM images of bimetallic nanoparticles are distinctly different from the monometallic counterparts and also the core-shell in near structure is visible in the bimetallic nanoparticles. CTAB in this reaction serves the purpose of a stabilizing agent in aqueous solution. Surfactants not only impart stability to the metal nanoparticles but also control the size and shape of the nanoparticles, and act against aggregation.[33-35]

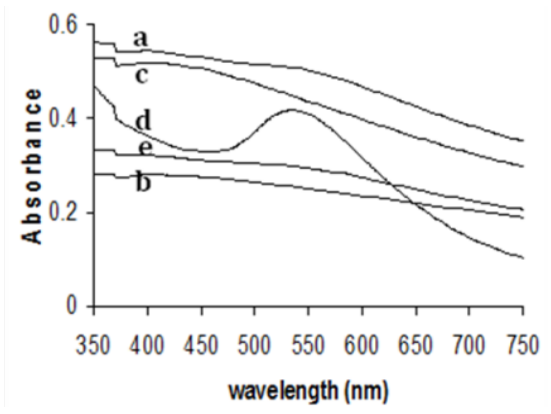

Figure 1. Typical UV-Vis spectra of metal nanoparticles with CTAB capping agent consisting of (a) 1:0.5, (b) 1:1, (c) $0.5: 1$ of the Au and Pd salts (d) monometallic $\mathrm{Au}$ and (e) Pd solution in aqueous medium at $25^{\circ} \mathrm{C}$

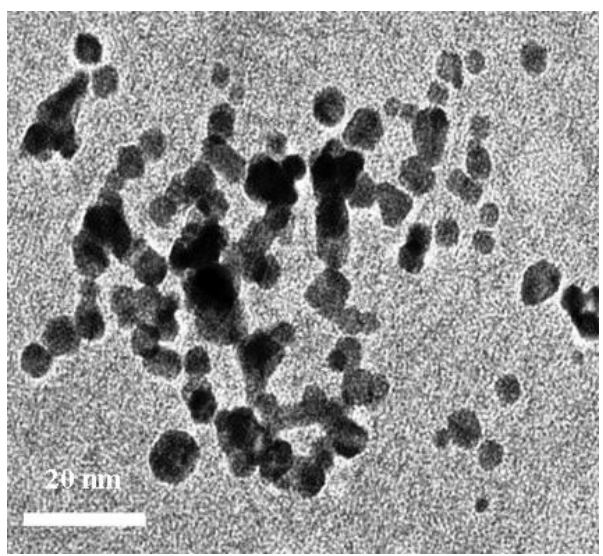

Figure 2. HRTEM images of the bimetallic nanoparticles (Au:Pd, 1:1)

The XRD patterns of single metallic Aunp, Pdnp and the 1:1 stoichiometry bimetallic Au-Pd is given in Figure 3. It may be seen that, the crystalline phases of monometallic nanoparticles are also present in the bimetallic nanoparticles. In Table 1, the particles size measurement values are given for mono and bimetallic nanoparticles also. It can be seen that the mono and bimetallic nanoparticles are mono disperse with an average size of 5.2, 5.8, $9.0 \mathrm{~nm}$ corresponding to mono metallic $(\mathrm{Au}, \mathrm{Pd})$ and bimetallic $(\mathrm{Au}-\mathrm{Pd})$ nanoparticles respectively. The diffractograms of the bimetallic combinations generally show broad bands while their monometallic counterparts exhibit fairly sharp bands. The particle sizes of the mono and bimetallic combinations determined from the XRD spectra correlate well with the sizes 
obtained from TEM measurements.

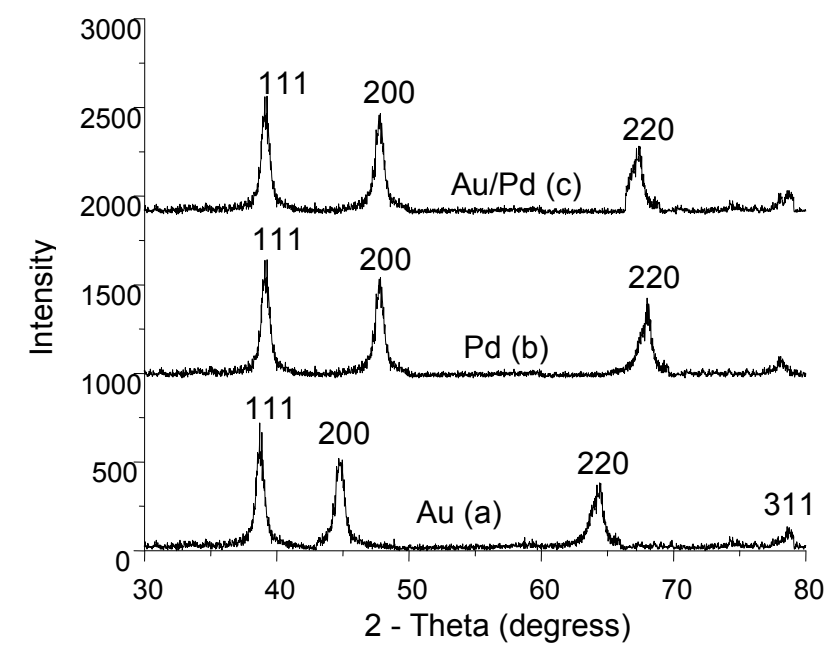

Figure 3. XRD pattern of mono metallic $(a, b)$ and bimetallic nanoparticles (c)

Table 1. The XRD and SEM-EDX spectral data of mono and bimetallic nanoparticles

\begin{tabular}{|c|c|c|c|}
\hline $\begin{array}{c}\text { Initial atomic } \\
\text { Composition } \\
\mathrm{Au}: \mathrm{Pd}\end{array}$ & $\begin{array}{c}\text { Particles } \\
\text { sizes(nm) }\end{array}$ & $\begin{array}{c}\text { Structure of } \\
\text { nanoparticles }\end{array}$ & $\begin{array}{c}\text { atomic composi- } \\
\text { tion in nanoparti- } \\
\text { cle Au:Pd }\end{array}$ \\
\hline $1: 0$ & 5.2 & $\mathrm{fcc}$ & $0.94: 0$ \\
\hline $0: 1$ & 5.8 & $\mathrm{fcc}$ & $0: 0.98$ \\
\hline $1: 1$ & 9.0 & $\mathrm{fcc}$ & $0.94: 0.98$ \\
\hline $1: 0.5$ & 8.8 & $\mathrm{fcc}$ & $0.96: 0.48$ \\
\hline $0.5: 1$ & 9.2 & $\mathrm{fcc}$ & $0.42: 0.90$ \\
\hline
\end{tabular}

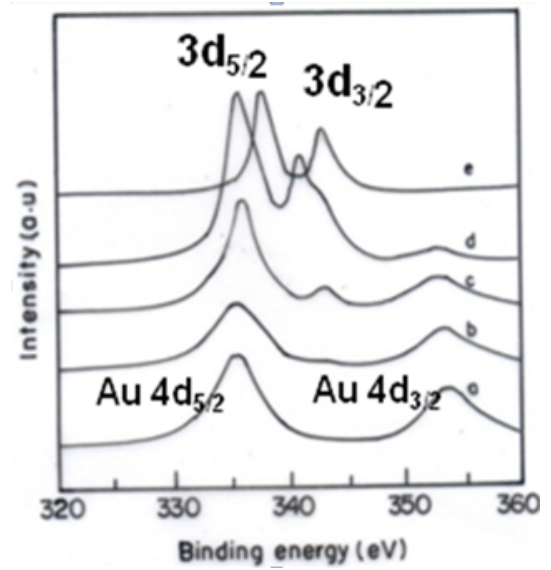

Figure 4. XPS spectra of mono metallic $(a, b)$ and bimetallic nanoparticles (c)

\subsection{X-ray Photoelectron Spectroscopy Analysis}

XPS spectroscopy has been widely used to study the surface chemistry of metal nanoparticles. To further investigate the oxidation states of the elements and the composition distribution in a particle, the compositions in the outer part of bimetallic nanoparticles were measured. The XPS spectra of the $\mathrm{Au} 4 \mathrm{f}$ and $\mathrm{Pd} 3 \mathrm{~d}$ regions of the CTAB-capped nanoparticles are shown in Figure 4. A doublet at (B.E) 335.4 and $354.0 \mathrm{eV}$ corresponding to $\mathrm{Au}-4 \mathrm{~d}$, in the respective multimetallic system represents metallic state of the colloids (Fig.4a). In Fig.4e, a doublet of Pd-3d at 338.0 and $344.0 \mathrm{eV}$ with two narrow peaks is further evidence that palladium is present in its metallic state. No other oxidation states of palladium were detected in the sample. The latter decreased with the increase of $\mathrm{Au}-\mathrm{Pd}$ ratio and should result from the formation of Pd-CTAB. Pd-CTAB should also be formed on the surface of Pd and Au-Pd particles (Fig.4b,c,d). According to the intensities of XPS peaks, the elemental ratios of Au-Pd in the outer part of bimetallic nanoparticles could be obtained. It was obvious that the Pd atoms were enriched in the outer part of Au-Pd bimetallic nanoparticles, suggesting a structure of incomplete Au-core-Pd-shell. This was consistent with the observation of UV-vis absorption spectra. The control of composition distribution by feeding composition should be interesting and important for the preparation of catalysts.
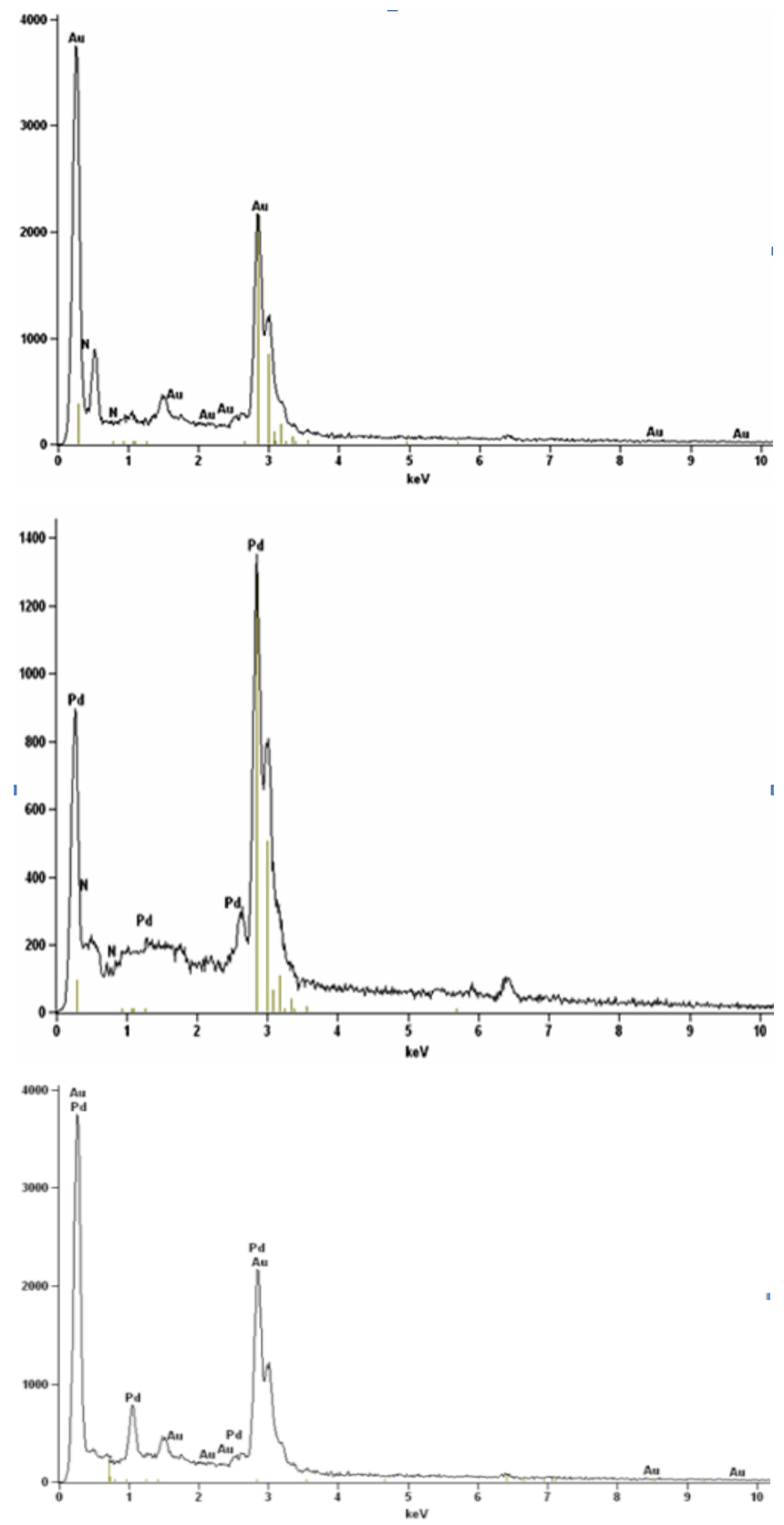

Figure 5. SEM-EDX spectra of mono metallic $(a, b)$ and bimetallic nanoparticles (c) 
Energy dispersive X-ray analyses are also conducted by focusing the electron beam on several different selected regions on each sample (each region area $1 \mu \mathrm{m} \times 1 \mu \mathrm{m}$ ) of the unsupported Au-Pd nanoparticles. An EDX spectrum of Au-Pd nanoparticles is shown in Figure 5. The compositions of Au-Pd bimetallic nanoparticles in different regions are in close agreement and no significant deviations initial and final synthesized atomic composition in bimetallic $\mathrm{Au}: \mathrm{Pd}$ nanoparticles nanoparticle.

Figure 6 shows FT-IR spectra of pure CTAB, Aunp, Pdnp and Au-Pdnp respectively. In the spectral region 3550 $-2500 \mathrm{~cm}^{-1}$, the $\mathrm{C}-\mathrm{H}$ symmetric and asymmetric stretching vibration frequency modes at 2850 and $2920 \mathrm{~cm}^{-1}$ seen for pure CTAB appear at 2862 and $2940 \mathrm{~cm}^{-1}$ for Aunp-CTAB, 2858 and $2944 \mathrm{~cm}^{-1}$ for Pdnp-CTAB while at 2872 and $2958 \mathrm{~cm}^{-1}$ for Au-Pdnp-CTAB nanoparticle systems respectively. The doublet at 1462 and $1472 \mathrm{~cm}^{-1}$ for pure CTAB may be attributed to the $\mathrm{CH}_{2}$-scissoring modes of vibrations, which are broad with lesser intensity and shifted to lower wave numbers in the nanoparticles. These data clearly indicate that the binding of CTAB to mono and bimetal nanoparticles.

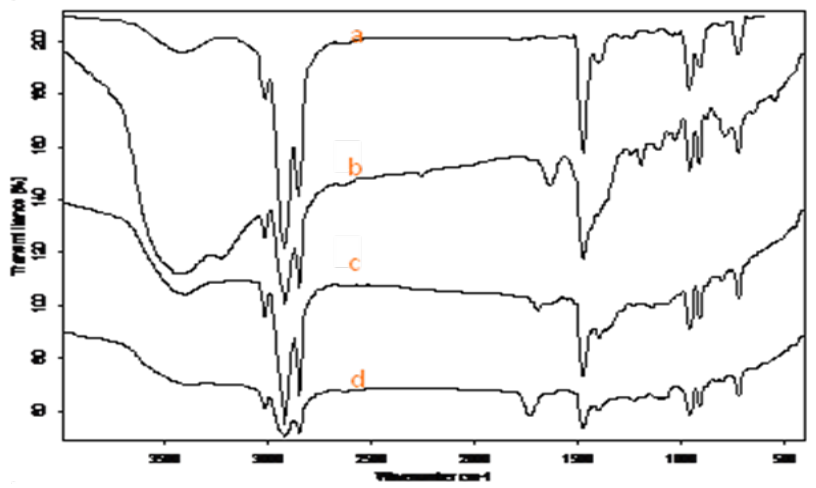

Figure 6. FTIR spectra of (a) CTAB, (b) Aunp (c) Pdnp and (d) Au/Pdnp, in $\mathrm{KBr}$ pellet at $25^{\circ} \mathrm{C}$

\subsection{Catalytic Application of Hydrogenation of 4-Nitrophenol}

Recent reports showed that the bimetallic colloids are constituted of two distinct metals. Such compounds often showed better activity and selectivity compared to mono metallic colloids. In the present work, the 4-Nitrophenol reduction reaction was monitored by UV-Vis spectroscopy and the product yields were determined by gas chromatography. Each catalytic reaction was carried out in at least two runs. Both plots reveal that using $0.5 \mathrm{~mol} \%$ of bimetallic (1:1) was sufficient to catalyze the reduction reaction with conversion and product yield of $\geq 96 \%$. We have successfully fabricated the first example of surfactant stabilized bimetallic nanoparticles using wet chemical method of mixtures consisting of $\mathrm{Au}$ and $\mathrm{Pd}$ colloids. Figure 7 shown that, the present Au-Pd bimetallic nanoparticles demonstrated good catalytic properties on the product formation test platform, performing better than mono metallic palladium and gold nanoparticles. When the recovered catalyst is
UV-Visible spectra recoated, the SPR peaks are not as prominent as in the first cycle.

The turnover frequency of the catalysts has been measured by recovering the bimetallic nanoparticles precipitates are subjected 4-Nitro phenol reduction reaction catalysis. It is found that $99,92,86$ and 46 percent product yields existed corresponding to fresh $1^{\text {st }}, 2^{\text {nd }}$ and $3^{\text {rd }}$ reuses respectively. Catalyst reuse in the fourth time showed that, the reaction did not proceed. The metallic nanoparticles are definitely much less catalytically active during the second cycle of the reduction reaction. The reason is due to a lower amount of nanoparticles present in the solution if due to precipitation of larger nanoparticles. Another possibility is that if the number density has not changed, but only the size is getting smaller, that the smaller particles might not be as catalytically active as the larger particles. As a result, the lower catalytic activity observed during the second cycle is due to a lower amount of nanoparticles present in the solution due to larger nanoparticles aggregating and precipitating out of solution. In addition, surface poisoning by the products could be another reason. After the completion of the reduction reaction, the bimetallic nanoparticles catalyst and the products are correspondingly separated into the $\mathrm{pH}$ responsive aqueous and organic phases respectively.

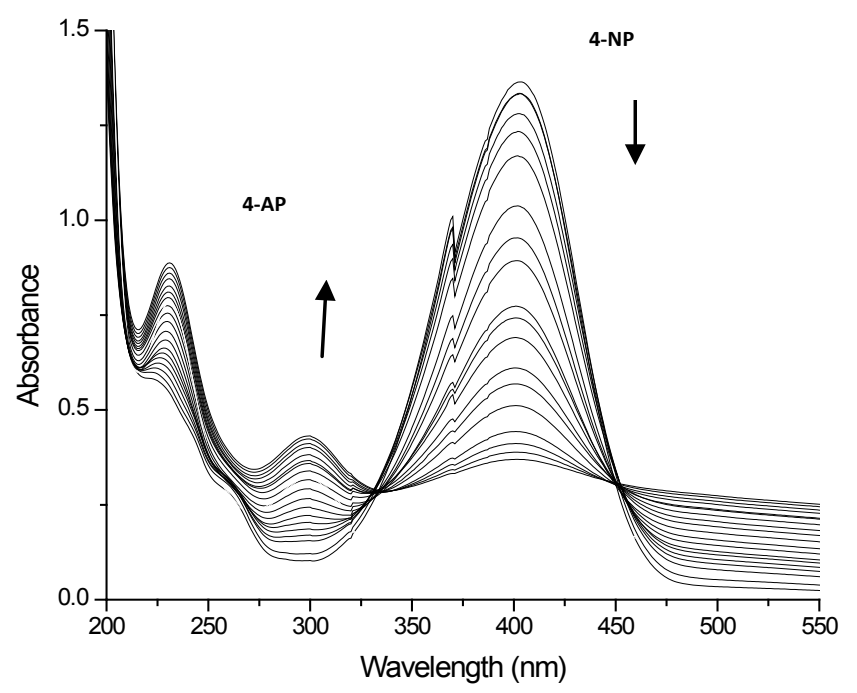

Figure 7. Time variance reduction of 4-Nitrophenol in Au-Pd bimetallic nanoparticles

\section{Conclusions}

In conclusion, we have synthesized a novel family of Au-Pd core-shell bimetallic nanoparticles from the wet chemical method of metal-surfactant complexes has been reported. Surface plasmon peaks in UV-Vis spectroscopy has been conformed the formation of mono and bimetallic nanoparticles. The particle size results from HRTEM and XRD agree well with each other. SEM-EDX spectra confirmed the presence of the two metals. XPS peaks, the elemental ratios of Au-Pd in the outer part of bimetallic nanoparticles could be obtained. XPS spectroscopy has been 
widely used to study the surface chemistry of metal nanoparticles that the Pd atoms were enriched in the outer part of Au-Pd bimetallic nanoparticles, suggesting a structure of Au-core-Pd-shell structure. Their catalytic activities were tested on the hydrogenation reaction between $\mathrm{NaBH}_{4}$ and 4-Nitrophenol reduction was studied spectrophotometrically. These novel palladiums containing bimetallic nanoparticles are air- and moisture-stable, and the reaction can be readily conducted under aerobic conditions. Bimetallic Au-Pd nanoparticle catalytic reduction of 4-Nitrophenol reaction produced better results than mono catalysts. The high catalytic activity of bimetallic nanoparticles is probably due to the sequential electronic effect between elements in a particle. More detailed investigations of nanoparticle structure effects on the catalytic activity and their applicability in other synthetic transformations are currently under investigation.

\section{ACKNOWLEDGMENTS}

The authors thank financial support to the Department from DST-FIST.

\section{REFERENCES}

[1] Shiho Tokonami, Nobuyasu Morita, Kanako Takasaki, and Naoki Toshima, J. Phys. Chem. C, 2010, 114 (23), 10336

[2] Serpell, J. Christopher, Cookson, James; Ozkaya, Dogan; Beer, Paul D. Nature Chemistry 2011, 3(6), 478

[3] M. J. Hostetler, C. J. Zhong, B. K. H. Yen, J. Anderegg, S. M. Gross, N. D. Evans, M. Porter and R. W. Murray, J. Am. Chem. Soc. 1998, 120, 9396

[4] Dingsheng Wang and Yadong Li, Adv. Mater. 2011, 23, 9, 1044

[5] M. C. Daniel and D. Astruc, Chem. Rev. 2004, 104, 293

[6] I. Srnova-Sloufova, B. Vlckova, Z. Bastl and T. L. Hasslett, Langmuir 2004, 20, 3407

[7] T. Shibata, B. A. Bunker, Z. Zhang, D. Meisel, C. F. Vardeman and J. D. Gezelter, J. Am. Chem. Soc. 2002, 124, 11989

[8] M. Moskovits, I. Srnova-Sloufova and B. Vlckova, J. Chem. Phys.2002, 116, 10435

[9] C. J. Zhong and M. M. Maye, Adv. Mater. 2001, 13, 1507

[10] J. S. Bradley, In Clusters and Colloids; Schmid, G., Ed.; Wiley- VCH: Weinheim 1994; Chapter 6

[11] L.N. Lewis, Chem. Rev. 1993 93, 2693
[12] G. Schmid (eds), Cluster and Colloids: From Theory to Applications, (VCH, New York, 1994)

[13] N. Toshima and T. Yonezawa, New J. Chem. 1998, 1179.

[14] J.H. Sinfelt, Accounts Chem. Res. 1987, 20, 134

[15] M. Brust, M. Walker, D. Bethell, D. J. Schiffrin and R. Whyman, J. Chem. Soc., Chem. Commun. 1994, 801

[16] M. J. Hostetler, J. E. Wingate, C. J. Zhong, J. E. Harris, R. W. Vachet, M. R. Clark, J. D. Londono, S. J. Green, J. J. Stokes, G. D. Wignall, G. L. Glish, M. D. Porter, N. D. Evans and R. W. Murray, Langmuir 1998, 14, 17

[17] Christopher J. Serpell, James Cookson, Dogan Ozkaya and Paul D. Beer, Nature Chemistry. 2011, 3, 478

[18] S. Ajaikumar, J. Ahlkvist, W. Larsson, A. Shchukarev, A. R. Leino, K. Kordas and J. P. Mikkola. Applied Catalysis A, General. 2011, 392, (1-2), 11

[19] M. Valden, X. Lai and D. W. Goodman, Science 1998, 281, 1647

[20] G. C. Bond and D. T. Thompson, Gold Bull. 2000, 33, 41

[21] G. C. Bond, Catal. Today 2002, 72, 5

[22] J. Santhanalakshmi. P. Venkatesan. J. Nanopar. Res. 2011, 13, 479

[23] M. J. Kim, H. J. Na, K. C. Lee, E. A. Yoo and M. Y. Lee, J. Mater. Chem. 2003, 13, 1789

[24] C. Fan and L. Jiang, Langmuir 1997, 13, 3059

[25] S. Link, and M. A. El-Sayed, J. Phys. Chem. B 1999, 103, 8410

[26] S. Link, Z. L. Wang and M. A. El-Sayed, J. Phys. Chem. B 1999, 103, 3529

[27] Y. S. Shon, G. B. Dawson, M. Porter and R. W. Murray, Langmuir 2002, 18, 3880

[28] B. R. Gonzalez, A. S. Iglesias, M. Giersig and L. M. L. Marzan, Faraday Discuss. 2004, 125, 133

[29] D. H. Chen and C. J. Chen, J. Mater. Chem. 2002, 12, 1557

[30] Y. M. Chung and H. K. Rhee, Catal. Lett. 2003, 85 (3-4) 159

[31] Y. M. Chung and H. K. Rhee, J. Mol. Catal. A: Chemical 2003, 206, 291

[32] Y.M. Chung and H. K. Rhee, J. Colloid Interf. Sci. 2004, 271, 131

[33] J. J. Gao, J. Fu, C. Lin, J. Lin, Y. Han, X. Yu and C. Pan. Langmuir, 2004, 20, 9775

[34] X. S. Wang, H. Wang, N. Coombs, M.A. Winnik and I. Manners. J. Am. Chem. Soc. 2005, 127, 8924

[35] R. C. Doty, T.R. Tshikhudo, M. Brust and D.G. Fernig, Chem. Mater. 2005, 17, 4630 